\title{
Classification of EMG signal for multiple hand gestures based on neural network
}

\author{
Mohd Azlan Abu', Syazwani Rosleesham², Mohd Zubir Suboh³ ${ }^{3}$, Mohd Syazwan Md Yid", \\ Zainudin Kornain ${ }^{5}$, Nurul Fauzani Jamaluddin ${ }^{6}$ \\ 1,2,3,4 Universiti Kuala Lumpur British Malaysian Institute, Malaysia \\ ${ }^{5}$ Royal Commission Yanbu Colleges and Institutes, Yanbu Alsinaiyah, Saudi Arabia \\ ${ }^{6}$ RT Technology Sdn Bhd, Malaysia
}

\begin{tabular}{l}
\hline \hline Article Info \\
\hline Article history: \\
Received Apr 19, 2019 \\
Revised Jun 20, 2019 \\
Accepted Jul 4, 2019 \\
\hline
\end{tabular}

\section{Keywords:}

Electromyography

EMG signals

Hand gestures

Medical electronics

Neural network

\begin{abstract}
This paper presents the classification of EMG signal for multiple hand gestures based on neural network. In this study, the Electromyography is used to measure the muscle cell's electrical activities which is commonly represented in a function time. Every muscle has their own signals, which was produced in every movement. Surface electromyography (sEMG) is used as a non-invasive technique for acquiring the EMG signal. The development of sensors' detection and measuring the EMG have been improved and have become more precise while maintaining a small size. In this paper, the main objective is to identify the hand gestures based on: (1) Cylindrical Grasp, (2) Supination (Twist Left), (3) Pronation (Twist Right), (4) Resting Hand and (5) Open Hand that are predefined by using Arduino IDE, CoolTerm software and Microsoft Excel before using artificial neural network for classifying purposes in MATLAB. Finally, the extraction of the EMG patterns for each movement went through features extraction of the signals which is used to train the classifier in MATLAB to classify signals in the neural network. The features extracted are using mean absolute value (MAV), median, waveform length (WL) and root mean square (RMS). The Artificial Neural Network (ANN) produced accuracy of $80 \%$ for training and testing for 10 hidden neurons layer.
\end{abstract}

Copyright $\odot 2020$ Institute of Advanced Engineering and Science. All rights reserved.

\section{Corresponding Author:}

Mohd Azlan Abu,

Universiti Kuala Lumpur British Malaysian Institute,

Batu 8, Jalan Sg Pusu, 53100 Gombak, Selangor, Malaysia.

Email: mohdazlan@unikl.edu.my

\section{INTRODUCTION}

Bioelectrical signals from human body are extensively studied and applied into various researches everywhere in this world. However, the purpose of using these signals for the field of information technology is still new and developing [1]. One of the human bioelectrical signals is myoelectric signal or electromyogram (EMG) signal which is used in this study [2]. Myoelectric signal or EMG signal is a motor action potential (MUAP) [3]. This signal is like electrical activity of the bundle of muscle cells which originates from the activity of the muscle as a response to the nerve's stimulation in the contraction of muscle fibers in the body. The electrical signal makes detection of muscular activity available for study which then provides an important role in hand gesture recognition. The signal is attained by using needle electrode or by surface electrodes (sEMG). The sensors attached to the skin surface on top of the bundle of muscles is known as surface electromyography (sEMG) $[4,5]$.

Researchers are very keen on using sEMG because this method is a non-invasive way to acquire signals and have been using it extensively in various field in current time, for example in prostheses development, rehabilitation, identifying muscle injury and in classifying several neuromuscular diseases [6]. 
Identification of surface electromyography (sEMG) signal gives the most powerful source in control signal to develop a better myoelectric prosthetic arms/hands [7]. Hand gestures can be recognized through this EMG signal, in the intention of creating a hand prosthesis that is more affective and more natural [8].

The target for this study is to discriminate between five movements, namely resting hand, open hand, cylindrical grasp, lateral grasp and wrist pronation and supination. The purpose of this approach is for a much natural grasping movement that later on will allow the wrist to move together with the object for a better grasping [9]. The findings of this study will be useful for future use of the society and commercial considering that there are many people out there are looking for a better prosthetics arms, a better technology to make them feel like they are using real arms. In this research, in hope that by the classification of EMG signals for multiple hand gesture will help in producing a better prosthetics arm.

According to [10], the journal discussed on ways to improve the effectiveness of hand prosthetic control in much natural way by using electromyography (EMG) signals. The study found out that there are five movements that are important for grasping where the position is intended for wrist-hand mobility. The researchers built a simple acquisition and analysis of EMG signals that can be used into prosthetic hand. They also finalized that forearm of the hand is chosen for electrodes positioning for signal collection while to run the system, Artificial Neural Networks (ANN) and wavelet features are used. They gathered that using their method, the accuracy is nearly $90 \%$.

In journal [11], the identification of hand gestures from electromyography (EMG) where the electrical activity of the muscles are denoted from function of time through Artificial Neural Network (ANN) and LabVIEW. The researchers use the method for classification. Four hand gestures are chosen for classification. They studied the EMG signals formed which extracted from each movement of hand gestures, before training the neural network for classification to be done with features like mean absolute value, root mean square, variance, standard deviation, mean frequency, zero crossing and slope sign change.

From [12], the study is also in the intention towards effectiveness of hand prosthetic. The researcher in this study uses forearm muscles to for extraction of EMG signals while for classification of the signal, the researcher use Wavelet transform first before using the time-frequency featured in Artificial Neural Network (ANN). The researcher chose five hand gestures to have the EMG signals collected with different placement of electrodes on the forearm areas, thus they claimed that this improved the number of functions that a system can operate, making previous journal [9] as a reference as it uses two electrodes in getting the EMG signals.

According to [13], the journal is to develop new classification of EMG signal method with more accuracy for pattern recognition through method of neural network model. The classification model is in MATLAB platform which has accuracy in classification an average $96.7 \%$ but researchers only choose 3 hand gestures for classification. The journal concluded that using neural network method turned out has high level of classification accuracy and very effective classifier.

In [14] journal, the electromyography (EMG) signal is taken through method of Wavelet transform and MATLAB tools which have accuracy in classification of hand gestures of $87 \%$ but the researchers only choose 2 hand gestures for classification. The signals received are de-noised in a parameter which then can be applied into calculation for basic prostheses hand design.

In another journal [15], six different hand gestured are picked to produce EMG signals than then will be extracted using MATLAB software and trained into classifier for further real-time validation which uses support vector machine (SVM) with round-based kernel function (RBF). The researchers aimed to create a system that test for pattern recognition of electromyography (EMG) signal, thus after developing the system, they concluded that their study is acceptable considering on the result from the training procedure and from online validation.

Other papers [16-20], they aimed to classify hand gestures by using EMG signals where seven features are extracted from it. For the hand gestures, four gestures are chosen. The researchers used a Feedforward Artificial Neural Network is used as classifier that trained to recognize the gestures which later used for human-robot interaction [21-25]. This paper present the classification of EMG signal for multiple hand gestures based on neural network using by using Arduino IDE, CoolTerm software and Microsoft Excel for data collection and finally, MATLAB is used for classification part using artificial neural network. The Artificial Neural Network (ANN) produced accuracy of $80 \%$ for training and testing for 10 hidden neurons layer.

\section{RESEARCH METHOD}

The MyoWare ${ }^{\mathrm{TM}}$ Muscle Sensor (AT-04-001) with Ag/Ag Cl electrode attached to subject muscle, an Arduino UNO board and also Arduino IDE, CoolTerm, Microsoft Office and MATLAB software are the components of this research study. Description about the hardware architecture and the software 
implementation are explained further in this chapter. Block diagram and flow chart show the flow of the data collection system to make it more understandable and visualized. Figure 1 shows the block diagram for this study. The block diagram starts with acquiring EMG signals from the assembly of MyoWare ${ }^{\mathrm{TM}}$ Muscle Sensor (AT-04-001) that used electrode pads attached onto subject and Arduino UNO board that is programmed to produce voltage output by Arduino IDE software. The data acquisition collected via CoolTerm software that work like Arduino IDE Serial Monitor is saved in Microsoft Excel folder as database of EMG signal. Feature extraction is done according to time and frequency domain in the Microsoft Excel as '.xlxs' file. The '.xlsx' file is then called into MATLAB for classification using Artificial Neural Network toolbox. Classification of the hand gestures and accuracy are the result in post processing.

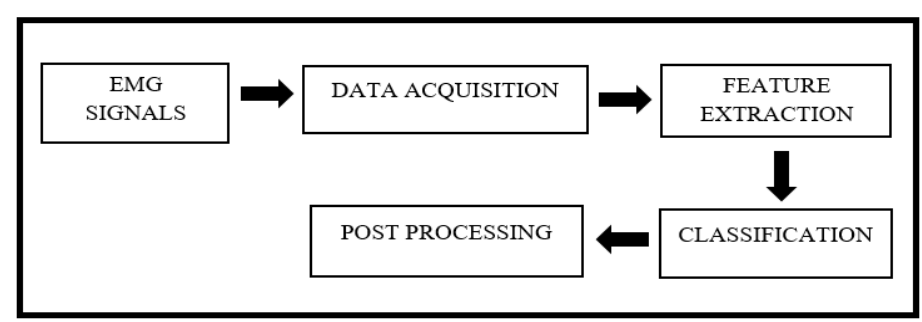

Figure 1. Block diagram of this study

\subsection{Type of Multiple Hand Gestures}

In this paper, the hand gestures recorded for EMG signals are shown in Figure 2 which are taken on the left arm of person with various poses according to the research done; (1) Cylindrical Grasp (2) Supination (Twist Left) (3) Pronation (Twist Right) (4) Resting Hand (5) Open Hand.

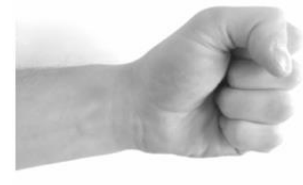

1) Cylindrical Grasp

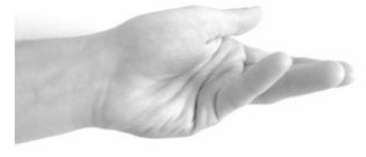

2) Supination (Twist Left)

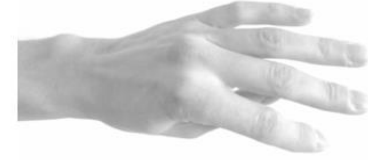

3) Pronation (Twist Right)

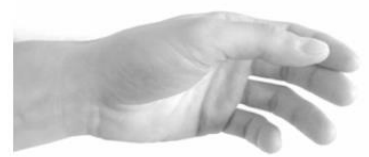

4) Resting Hand

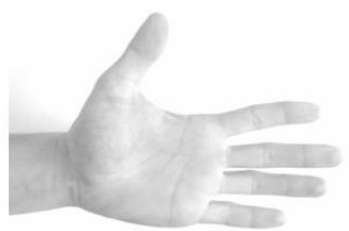

5) Open hand

Figure 2. Type of multiple hand gestures [15]

\subsection{Data Acquisition}

Two subjects, both females, aged 22 and 23 are recruited to perform the required hand gestures selected. The subjects are all normally limbed with no muscular or neurological disorders. During the process, subjects are seated on an armchair in order to let their arm be supported and fixed in one position. This is to avoid any effects of different limb movement on the EMG signals generated. The EMG signals are recorded by using left forearm muscles.

Five hand gestures which are (1) Cylindrical Grasp (2) Supination (Twist Left) (3) Pronation (Twist Right) (4) Resting Hand (5) Open Hand. The total number of signals collected is 1200 for each hand movement for 6 seconds. Attempt done for each movement is 10 time. Each attempt is done separately to avoid muscle fatigue that will affect the EMG signals generated. MyoWare ${ }^{\text {TM }}$ Muscle Sensor (AT-04-001) is placed at Brachioradialis and Flexor Carpi muscle as these two muscles are the major muscles involved in hand gestures (Jaramillo \& Benalcazar, 2017). 
CoolTerm software is used to save the analog voltage data of the EMG signals recorded by MyoWare ${ }^{\mathrm{TM}}$ Muscle Sensor (AT-04-001). The data is saved in Microsoft Excel according to each motion.

\subsection{Feature Extraction}

The EMG signals generated are an extensive information part that also can be redundant. To make the signal to be correctly exploited, the signal should be through feature extraction. Feature extraction is a process where the signal characteristics are decomposed and mixed to form a vector feature that comprises a total representation of the acquired signal [13].

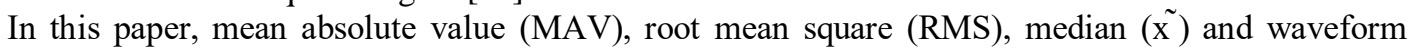
length (WL) provide acceptable classification. These are the 4 input features in this network. In the input feature, as each subject has 10 attempts, the total attempt to be send into the network is 20 . These 20 attempts are the feature extractions extracted from the analog voltage data collected via EMG sensor.

This process is carried out in Microsoft Office with 17 attempts of the input feature for training and 3 attempts are for testing. The '.xlsx' file is called to neural network toolbox in MATLAB for classification.

\subsection{Data Classification}

After the extraction of required features from the EMG signals, the feature extraction is put in a specific class in a way that can have an improvement in pattern recognition and in the interpretation. A classifier with proper speed, adequacy and ability to handle difference in the feature extractions values due to any outsider effects, are the important characteristics of interest [13].

The database signals in Microsoft Excel that have been arranged and processed into feature matrix are delivered to neural network model (NN-model) by using the neural network toolbox in MATLAB. Training, validating and testing are three stages for ANN data preparation. All are carried out following the respective percentage of $70 \%, 15 \%$ and $15 \%$. Every matrix row and column represent a signal and a feature accordingly. The hand gestures are categorized for target matrix in class number using binary code as follows:

MOTION 1 ans = [000]; MOTION 2 ans = [001]; MOTION 3 ans = [010]; MOTION 4 ans = [011]; MOTION 5 ans = [100];

The various classes were as follows:

a) Motion 1 Cylindrical Grasp

b) Motion 2 Supination (Twist Left)

c) Motion 3 Pronation (Twist Right)

d) Motion 4 Resting Hand

e) Motion 5 Open Hand

The features and target matrices are fed to the neural network system as input and target matrices. In Figure 3 shows the pattern recognition structure of the neural network. Number of hidden layers are specified while the system training is initiated. The hidden neurons are set to 10 and the output neurons is set to 3 which equals to number of elements in target vector.

For further classification, the recorded EMG signals were extracted with time and frequency domain which are mean absolute value (MAV), root mean square (RMS), median and waveform length (WL) that are taken from 50 samples per subject [16]. MAV is produced by calculating the absolute value of the signal's average as shown in (1). RMS is represented as a random amplitude modulated procedure in which Gaussian RMS is linked to the continuous and non-fatiguing contraction force [17] shown in (2). Median is acquired by separating the value of samples into two equal halves as shown in (3). WL is obtained by calculating the cumulative changes in amplitude from one-time sample to another over the entire time period shown in (4).

$$
\begin{aligned}
& \text { Mean Absolute Value, } M A V=\frac{1}{N} \sum_{n=1}^{N}\left|x_{n}\right| \\
& \text { Root Mean Square, } R M S=\sqrt{\frac{1}{N} \sum_{n=1}^{N} x^{2}(n)} \\
& \text { Median, } \tilde{x}=\left(\frac{n}{2}\right) \\
& \text { Waveform Length, } W L=\sum_{n=1}^{N}|x(n+1)-x(n)|
\end{aligned}
$$

In this study, back propagation (BP) is used by ANN for classification purpose. The network for training purpose is fed with input vectors and target vectors. For efficiency of the training purpose, the input 
feature vectors are normalized. By using gradient of performance function to minimize the performance, the BP will determine how to alter the weights.

ANN consists of 3-layers which are input layer, tan-sigmoid hidden layer and linear output layer. Each of the layer except input layer has a weight matrix W, a b and an output vector a. Input weights (IW) is the weight matrices that are connected to inputs, while layer weights (LW) is the weight matrices coming from the hidden layer outputs [12].

Back propagation (BP) used Lavenberg-Marquardt algorithm for training where the network is generalized for overfitting and it is done by dividing the training input data. $70 \%$ of input data is used for training while $15 \%$ of input data is used for validation and another $15 \%$ for testing. When the network is not satisfied by the results, it will train again with the last saved results. This happened is for improving the network performance and reducing the number of training time [11].

Lavenberg - Marquardt algorithm or known as damped least-squares method is designed to work specifically with loss functions that take the form of a sum of squared errors. The algorithm shown in (5) can work without computing exact Hessian matrix but can work with gradient vector and Jacobian matrix. This algorithm is set in the artificial neural network toolbox in MATLAB as shown in Figure 3.

$$
\text { Lavenberg }- \text { Marquardt, } x_{k+1}=x_{k}-\left[J^{T} J+\lambda J\right]^{-1} J^{T}
$$

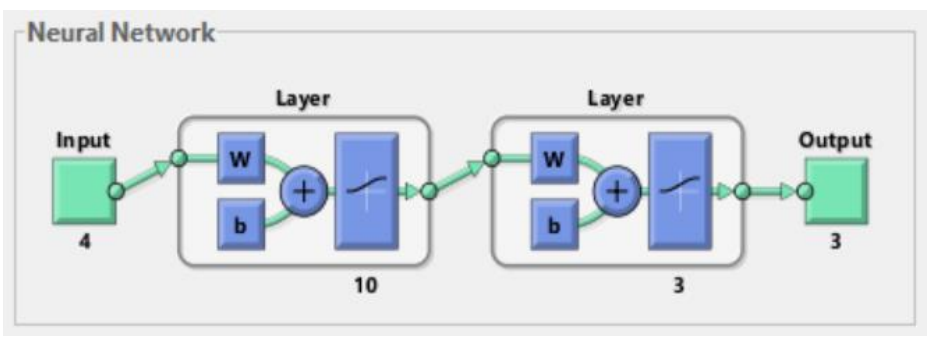

Figure 3. Pattern recognition and neural network structure

\subsection{Post Processing}

After data acquisition is extracted according to feature extraction selected, the classification is done by MATLAB. The results are in a categorized binary code as the hand gestures are inserted into matrix by using binary code and accuracy value from the ANN toolbox configuration. The results analysis will be discussed in next part.

\subsection{Flow of the Program}

The program starts by selecting the type of sensor and microcontroller to be used for data acquisition. Then, the five hand gestures selected for this study is performed, if there is no signal, troubleshooting is done. If there is signal that reached the voltage threshold level set, the data is collected into Microsoft Excel. After feature extraction is done, the extraction is trained and tested in MATLAB to acquire the classification of EMG signal results and the accuracy.

\section{RESULTS AND ANALYSIS}

After the neural network toolbox in MATLAB is trained with all of the features of every hand gestures, classification and accuracy can be obtained by testing the neural network. The number of hidden neuron set in the coding for this EMG signals classifier in neural network toolbox in MATLAB platform is 10 hidden neurons. After the classifier is trained with all of the features of every hand gestures, the result for accuracy is produced in the Command Window for each hidden neuron layer to see the system accuracy. Figure 4 shows the accuracy value in percentage for each number of hidden neuron for their training and testing.

Figure 5 shows a bar chart constructed from the classifier accuracy result for training and testing of the EMG signal feature extraction of the hand gestures selected in Command Window in MATLAB platform. All of the results to represent the accuracy training and testing in percentage result for every hidden neuron layer. In hidden neuron 1, the test accuracy and train accuracy is $40 \%$ while hidden neuron 2 test accuracy and train accuracy is $20 \%$. Hidden neuron 3 and hidden neuron 4 are both resulted $53.33 \%$. In hidden neuron 5 , the test and train accuracy is $66.67 \%$. Hidden neuron 6, the test accuracy and train accuracy is $73.33 \%$. 
In hidden neuron 7 , the test accuracy and train accuracy is $100 \%$ which shows that in this layer is the best accuracy found. The hidden layer 8 , the test accuracy and train accuracy is $60 \%$. Two last hidden neuron number 9 and 10 are both resulted in $80 \%$.

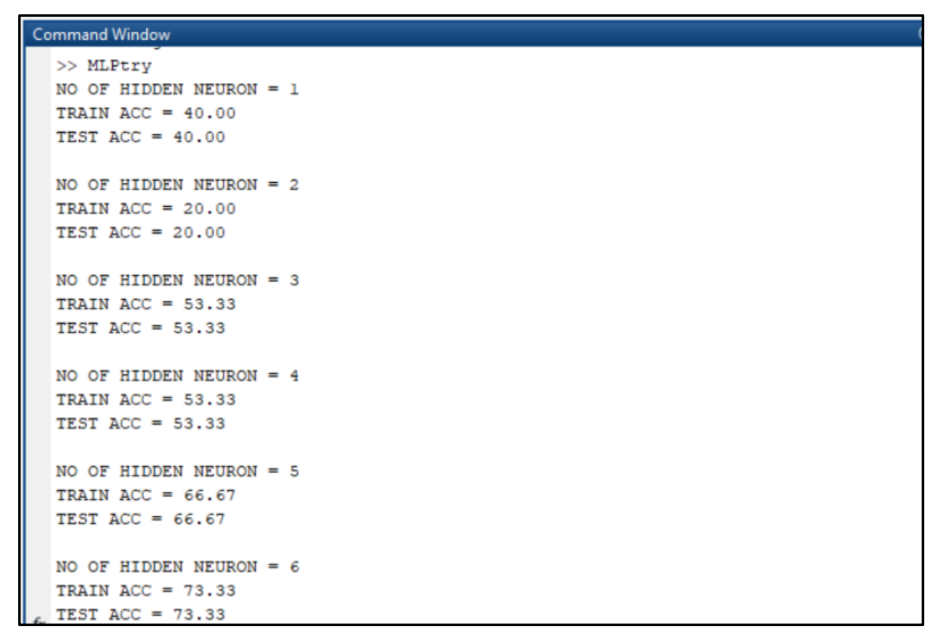

Figure 4. Command window from MATLAB

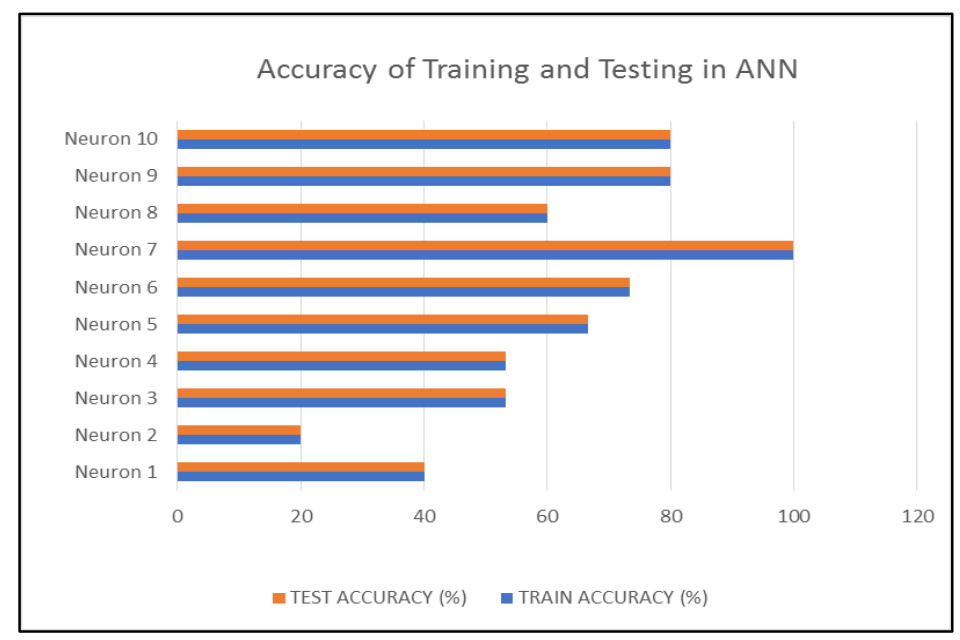

Figure 5. Accuracy result for 10 hidden neuron layers

The feature extractions of EMG signal are used to be classified by the neural network in MATLAB after the network has been trained with the motions. The classification of EMG signal for multiple hand gestures results are produced in binary code classes. The binary code classes set for every motions are ans = [000] for Cylindrical Grasp, ans = [001] for Supination (Twist Left), ans = [010] for Pronation (Twist Right), ans $=[011]$ for Resting Hand, ans $=[100]$ for Open Hand.

\section{CONCLUSION}

This paper presents the Classification of EMG Signal for Multiple Hand Gestures based on Neural Network for 5 wrist-hand gesture movements which are (1) Cylindrical Grasp (2) Supination (Twist Left) (3) Pronation (Twist Right) (4) Resting Hand (5) Open Hand. The objective is to use Artificial Neural Network (ANN) techniques to classify these signals have been achieved when each hand gesture features are put for classification in the Command Window of MATLAB platform. The results are according to the programmed input and target values are used to train and test the neural network. Thus, it can be concluded that the classification of EMG signals for multiple hand gestures by using Artificial Neural Network (ANN) is 
achieved without major flaws. The Artificial Neural Network (ANN) produced accuracy of $80 \%$ for training and testing for 10 hidden neurons layer. When cross-validations are completed, the average percentage of accuracy is also $80 \%$. This can be concluded that the classifier in MATLAB software by using Neural Network toolbox is able to train and test the EMG signals for the hand gesture successfully with the high accuracy value.

\section{ACKNOWLEDGEMENTS}

This work was partially supported by Short Term Research Grant (STRG) number STR17013 by Universiti Kuala Lumpur British Malaysian Institute, Gombak, Selangor.

\section{REFERENCES}

[1] Wan Mohd Bukhari Wan Daud, Abu Bakar Yahya, Chong Shin Horng, Mohamad Fani Sulaima, and Rubita Sudirman., "Features Extraction of Electromyography Signals in Time Domain on Biceps Brachii Muscle," International Journal of Modeling and Optimization, Vol 3, No 6, pp. 515-519, December 2013.

[2] Anita Pal, Ajeet Kumar Gautam, Yogendra Narain Singh, "Evaluation of Bioelectric Signals for Human Recognition,” Procedia Computer Science, Vol 48, 2015, pp 746-752, 2015.

[3] Chowdhury RH, Reaz MB, Ali MA, Bakar AA, Chellappan K, Chang TG., "Surface electromyography signal processing and classification techniques," Sensors (Switzerland), Vol 13(9), pp. 12431-12466, 2013.

[4] C. Castellini and P. V. D. Smagt, "Surface EMG in advanced hand prosthetics," Biological Cybernetics, vol. 100, no. 1 , pp. $35-47,2008$.

[5] S. Shin, J.-H. Lee, and S. H. Son, "A Survey of Approaches for Recognizing Hand Gestures Using EMG Signal," 2016 IEEE 22nd International Conference on Embedded and Real-Time Computing Systems and Applications (RTCSA), 2016.

[6] O. Iqbal, S. A. Fattah, and S. Zahin, "Hand movement recognition based on singular value decomposition of surface EMG signal," 2017 IEEE Region 10 Humanitarian Technology Conference (R10-HTC), pp. 827-842, 2017.

[7] R. M. G. Tello, T. Bastos-Filho, A. Frizera-Neto, S. Arjunan, and D. K. Kumar, "Feature extraction and classification of sEMG signals applied to a virtual hand prosthesis," 2013 35th Annual International Conference of the IEEE Engineering in Medicine and Biology Society (EMBC), Vol 4, No. 1, pp. 65-70, Feb 2014.

[8] I. Haider, M. Shahbaz, M. Abdullah, and M. Nazim, "Feature extraction for identification of extension and flexion movement of wrist using EMG signals," 2015 IEEE 28th Canadian Conference on Electrical and Computer Engineering (CCECE), pp. 792-795, 2015.

[9] S. A. Raurale, "Acquisition of EMG signals to recognize multiple Hand Gestures for Prosthesis Robotic Hand-A Review,” Int. J. Curr. Eng. Technol., pp. 65-70, 2014.

[10] G. Gini, M. Arvetti, I. Somlai, and M. Folgheraiter, "Acquisition and Analysis of EMG Signals to Recognize Multiple Hand Movements for Prosthetic Applications," Applied Bionics and Biomechanics, vol. 9, no. 2, pp. $145-155,2012$.

[11] E. H. Shroffe and P. Manimegalai, "Hand Gesture Recognition Based on Emg Signals Using Ann," IEEE Int. Conf. Consum. Electron. - Berlin, ICCE-Berlin, vol. 2016-Octob, no. 3, pp. 174-178, 2016.

[12] S. A. Raurale, "Acquisition of EMG signals to recognize multiple Hand Gestures for Prosthesis Robotic Hand-A Review," Int. J. Curr. Eng. Technol., pp. 65-70, 2014.

[13] Rami J. Oweis, Remal Rihani, Afnan Alkhawaja, "ANN-based EMG classification for myoelectric control," International Journal of Medical Engineering and Informatics, Vol 6, Issue 4, pp. 365, 2014.

[14] I. Haider, M. Shahbaz, M. Abdullah, and M. Nazim, "Feature extraction for identification of extension and flexion movement of wrist using EMG signals," Canadian Conference on Electrical and Computer Engineering, pp. 792-795, 2015.

[15] Konstantin Akhmadeev, Elena Rampone, Tianyi Yu, Yannick Aoustin, Eric Le Carpentier, "A testing system for a real-time gesture classification using surface EMG," IFAC-PapersOnLine, Volume 50, Issue 1, pp. 11498-11503, 2017.

[16] N. S. A. Sharawardi, Y. H. Choo, S. H. Chong, A. K. Muda, and O. S. Goh, "Single channel sEMG muscle fatigue prediction: An implementation using least square support vector machine," 2014 4th World Congress on Information and Communication Technologies, WICT 2014, 2014.

[17] M. G. B. Fonseca, A. G. S. Conceição and E. F. S. Filho, "Artificial neural networks applied to the classification of hand gestures using eletromyographic signals," 2017 Latin American Robotics Symposium (LARS) and 2017 Brazilian Symposium on Robotics (SBR), Curitiba, pp. 1-6, 2017.

[18] Mohd Zubir Suboh, Muhyi Yaakop, Mohd Shaiful Aziz Rashid Ali, Mohd Yusoff Mashor, Abdul Rahman Mohd Saad, Mohd Azlan Abu, Mohd Syazwan Md Yid, Aizat Faiz Ramli, "Portable heart valve disease screening device using electronic stethoscope," Indonesian Journal of Electrical Engineering and Computer Science, Vol. 15, No. 1, July 2019, pp. 122-132.

[19] Mohammed Lahraichi, Khalid Housni, Samir Mbarki, "Automatic foreground detection based on KDE and binary classification," Indonesian Journal of Electrical Engineering and Computer Science, Vol. 15, No. 1, July 2019, pp. 517-526, 2019. 
[20] Mohd Azlan Abu, Nurul Hazirah Indra, Abdul Halim Abd Rahman, Nor Amalia Sapiee, Izanoordina Ahmad, “ A study on Image Classification based on Deep Learning and Tensorflow," International Journal of Engineering Research and Technology, Volume 12, Issue 4 (2019), pp. 563-569, 2019.

[21] A. Ramli, H. Basarudin, M. A. Abu, M. Yaakop and M. I. Sulaiman, "FUSA: Fuzzy logic based clustering protocol for formation of uniform size clusters," 2017 International Conference on Engineering Technology and Technopreneurship (ICE2T), Kuala Lumpur, pp. 1-6, 2017.

[22] M. Z. Suboh, M. Yaakop, M. S. M. Yid, M. A. Abu and I. M. Sofi, "Heart valve disease screening system - PC based," 2017 International Conference on Engineering Technology and Technopreneurship (ICE2T), Kuala Lumpur, pp. 1-4, 2017.

[23] Z. Kornain, M. R. Abdullah and M. A. Abu, "Telemedicine system: Development of wireless healthcare units with GSM and Bluetooth link," 2012 IEEE Symposium on Industrial Electronics and Applications, Bandung, pp. 72-77, 2012.

[24] T. N. S. Tengku Zawawi, A. R. Abdullah, M.H. Jopri, T. Sutikno, N.M. Saad, R. Sudirman, "A Review of Electromyography Signal Analysis Techniques for Musculoskeletal Disorders," Indonesian Journal of Electrical Engineering and Computer Science, Vol. 11, No. 3, September 2018, pp. 1136-1146, 2018.

[25] M. Z. Suboh, M. Mashor, A. M. Saad, and M. Mohamed, "Classification of heart valve diseases using correlation analysis," 2008 th International Conference on Electrical Engineering, Computing Science and Automatic Control (CCE 2008), Mexico, pp. 173-176, 2008.

[26] M. B. Yaakop, I. A. A. Malik, Z. bin Suboh, A. F. Ramli and M. A. Abu, "Bluetooth 5.0 throughput comparison for internet of thing usability a survey," 2017 International Conference on Engineering Technology and Technopreneurship (ICE2T), Kuala Lumpur, pp. 1-6, 2017.

[27] M. A. Abu, M. F. Yaacob, A. F. Ramli, H. Basarudin, M. I. Sulaiman, M. Yaakop, I. M. Sofi, F. I. Romli, "Electrical appliances control system by using iOS," 2017 International Conference on Engineering Technology and Technopreneurship (ICE2T), Kuala Lumpur, pp. 1-5, 2017. 